\title{
Frecuencia de neoplasias en glándula mamaria de caninos diagnosticadas histopatológicamente en la Facultad de Medicina Veterinaria de la Universidad Nacional Mayor de San Marcos, periodo 2007- 2016
}

\author{
Frequency of neoplasms in mammary gland of canines diagnosed \\ histopathologically in the Faculty of Veterinary Medicine of the \\ Universidad Nacional Mayor de San Marcos, period 2007- 2016
}

\author{
Julisa Lipa C. ${ }^{1}$, Rosa Perales C. ${ }^{1,4}$, Viviana Fernández F. ${ }^{2}$, Gilberto Santillán A. ${ }^{1}$, \\ César Gavidia C. $^{3}$
}

\section{Resumen}

El objetivo de este estudio retrospectivo fue determinar la frecuencia de neoplasias en la glándula mamaria de caninos cuyo diagnóstico histopatológico se realizó en el Laboratorio de Histología, Embriología y Patología Animal de la Facultad de Medicina Veterinaria de la Universidad Nacional Mayor de San Marcos partir de los informes recopilados de 2007 hasta 2016, tomándose en cuenta las variables sexo, raza, edad, ubicación y diagnósticos histopatológicos. Las láminas fueron clasificadas según el sistema establecido por Goldschmidt et al. (2011). El total de procesos neoplásicos en caninos fue 1599, donde 359 correspondieron a neoplasias de glándula mamaria (22.4 \pm $\left.2.04 \% \mathrm{IC}_{0.95}\right)$. Las hembras fueron las más afectadas (98\%), mientras que la proporción de canes mestizos afectados fue de 32\%. El 68\% se presentó en razas puras, principalmente Cocker (13\%), Bóxer (5.9\%), Labrador (5.4\%) y Yorkshire (4.5\%). Canes de 10 años fueron los más afectados (17.7\%), siendo el estrato etario entre 9 y 12 años el más afectado (49.3\%). El 60.6\% de las neoplasias mamarias se presentaron en la cuarta y quinta glándula mamaria. El 94.7\%, de los tumores mamarios presentaron características histológicas malignas, siendo el carcinoma papilar quístico el tipo histopatológico más frecuente (42.8\%), seguido por carcinoma tubular (13.9\%).

Palabras clave: tumores; neoplasias mamarias; frecuencia; caninos; histopatología

\footnotetext{
${ }^{1}$ Laboratorio de Histología, Embriología y Patología Animal, Facultad de Medicina Veterinaria, Universidad Nacional Mayor de San Marcos, Lima, Perú

${ }^{2}$ Clínica de Animales Menores, Facultad de Medicina Veterinaria, Universidad Nacional Mayor de San Marcos, Lima, Perú

${ }^{3}$ Laboratorio de Medicina Veterinaria Preventiva, Facultad de Medicina Veterinaria, Universidad Nacional Mayor de San Marcos, Lima, Perú

${ }^{4}$ E-mail:rperalesc@unmsm.edu.pe
}

Recibido: 9 de octubre de 2018

Aceptado para publicación: 20 de abril de 2019 
The aim of this retrospective study was to determine the frequency of neoplasms in the mammary gland of canines whose histopathological diagnosis was made in the Animal Histology, Embryology and Pathology Laboratory of the Faculty of Veterinary Medicine of the Universidad Nacional Mayor de San Marcos (Lima, Peru). Reports were collected from 2007 to 2016, considering the variables sex, breed, age, location and histopathological diagnoses. The histological slides were classified according to Goldschmidt et al. (2011). The total of neoplastic processes in canines was 1599, where 359 corresponded to mammary gland neoplasms $\left(22.4 \pm 2.04 \% \mathrm{IC}_{0.95}\right)$. The females were the most affected (98\%), while the proportion of mestizo dogs affected was $32 \%$ while $68 \%$ corresponded to purebreds, mainly Cocker (13\%), Boxer (5.9\%), Labrador (5.4\%) and Yorkshire (4.5\%). Canines of 10 years old were the most affected $(17.7 \%)$, and the 9 and 12 years old was the most affected stratum age (49.3\%). In addition, $60.6 \%$ of mammary neoplasms occurred in the fourth and fifth mammary glands and $94.7 \%$ of mammary tumors presented malignant histological features, with cystic papillary carcinoma being the most frequent histopathological type (42.8\%), followed by tubular carcinoma $(13.9 \%)$.

Key words: tumors; mamary neoplasms; frequency; dogs; histopathology

\section{INTRODUCCIÓN}

La incidencia de neoplasias en animales de compañía ha presentado un aumento en los últimos años debido a múltiples razones, entre las que destacan la mayor longevidad de los caninos y la mayor preocupación de los propietarios por el bienestar de sus mascotas (Withrow y Vail, 2009).

Las neoplasias de glándula mamaria en perras presentan una incidencia más alta respecto a otras especies domésticas y tres veces más elevada que en humanos. Esto ha sido relacionado con los cambios hormonales de los ciclos estrales independientes de gestación (Núñez y Montes de Oca, 2012), de allí que los tumores mamarios son las neoplasias más frecuentes en perras sin esterilizar (Salas et al., 2015). Estos llegan a representar el $50 \%$ o más de todos los tumores en hembras, mientras que en machos son menos del 1\% (Dobson y Duncan, 2014; Withrow y Vail, 2009). Estudios previos del Laboratorio de Histología, Embriología y Patología Animal (LHEPA) de la Facultad de Medicina Veterinaria (FMV) de la Universi- dad Nacional Mayor de San Marcos (UNMSM), Lima, muestran la importancia de esta patología (Chau et al., 2013).

Los sistemas de clasificación histológicos utilizados para neoplasias y displasias mamarias en perras fueron publicados en 1974 (Hampe y Misdorp,1974) y modificados en 1999 (Misdorp et al., 1999). Posteriormente, en 2011 se propuso una nueva clasificación de los tumores mamarios en la perra (Goldschmidt et al., 2011). Por lo expuesto, el objetivo del presente estudio fue actualizar la clasificación de los diagnósticos histopatológicos y realizar una recopilación de la frecuencia de estos casos en la FMVUNMSM y sus variables como predisposición racial, edad, malignidad y localización a fin de proporcionar información epidemiológica a la clínica veterinaria.

\section{Materiales y MéTOdos}

Se realizó la recopilación de protocolos y evaluación de láminas histopatológicas del periodo comprendido entre enero de $2007 \mathrm{y}$ 
diciembre de 2016 del LHEPA de la FMVUNMSM, Lima, Perú. La información registrada incluyó el número de caso, raza (pura o mestiza), edad (0-4, 5-8, 9-12, >12), ubicación de las glándulas mamarias afectadas (torácica craneal, torácica caudal, abdominal craneal, abdominal caudal e inguinal), número de neoplasias mamarias (única o múltiples) y el diagnóstico histopatológico, según la clasificación de Goldschmidt et al. (2011).

Los datos obtenidos en el LHEPA se basan en tres aspectos: comportamiento, estirpe y morfología. Esta información fue recategorizada según la clasificación de Goldschmidt et al. (2011).

\section{Resultados}

En el periodo de 10 años (2007-2016) del estudio se recopilaron 1599 procesos neoplásicos en caninos, de los cuales el 22.4 $\pm 2.04 \% \mathrm{IC}_{0.95}(359 / 1599)$ correspondieron a neoplasias de glándula mamaria. De estos casos, 356 casos registraron el sexo, siendo 349 casos en hembras y 7 en machos. Asimismo, el $68 \%$ de los pacientes pertenecían a razas puras mientras que el $32 \%$ era de origen mestizo. Las razas más frecuentes fueron Cocker Spaniel 13\% (46/353), Bóxer $5.9 \%(21 / 353)$, Labrador 5.4\% (19/353), Yorkshire $4.5(16 / 353)$ y Shih Tzu $4.2 \%$ $(15 / 253)$, las cuales constituyen el $65 \%$ del total de casos.

El diagnóstico histopatológico (que incluye el comportamiento, estirpe celular y morfología) de los 359 protocolos incluyó 570 láminas histopatológicas de neoplasias de glándula mamaria. Al realizar la recategorización de los diagnósticos de las 30 (5.3\%) neoplasias benignas y $540(94.7 \%)$ neoplasias malignas mediante la clasificación de Goldschmidt et al. (2011), se identificó como la neoplasia maligna más frecuente al carcinoma $(86.3 \%)$, seguida por las neoplasias mesenquimales que representaron el 6.1,
(33/540). La neoplasia benigna más frecuente fue el tumor mamario mixto benigno con $1.8 \%$ (Cuadro 1 ).

La mayor frecuencia de presentación de neoplasias ocurre a los 10 años (62 casos, 17.7\%), seguido del grupo de 8 años (49 casos, 14\%). El estrato etario entre $9 \mathrm{y}$ 12 años fue el que presentó la mayor cantidad de casos $(173,49.3 \%)$, como se observa en la Figura 1, siendo el rango de edades de presentación entre 1 y 17 años.

El tipo celular que se afecta con más frecuencia es de origen epitelial maligno. Los tumores mamarios mixtos malignos se presentaron en un rango de edad entre los 6 y 14 años. Además, se observó una baja presentación en los caninos jóvenes menores a 4 años, y una curva ascendente de casos hasta los 12 años.

Con relación al número de neoplasias mamarias, $235(65 \%)$ presentaron un tumor en la cadena mamaria, mientras que 124 (35\%) presentaron múltiples tumores en diferentes glándulas mamarias. Aproximadamente el $60 \%$ de neoplasias se presentó en la cuarta y quinta glándula mamaria, se puede observar en el Figura 2 que las glándulas mamarias caudales son las más afectada, especialmente la glándula mamaria inguinal la que presenta neoplasias con mayor frecuencia.

\section{Discusión}

El 22.4\% de frecuencia de neoplasias de glándula mamaria fue similar a estudios previos realizados en Perú. Así, Orbegoso (1993) reportó 20.2\% (100/494) en casos entre 1973 y 1992 y Chau et al. (2013) registró el 18.1\% (204/1128) entre $1994 \mathrm{y}$ 2006. Asimismo, se puede observar un incremento en la frecuencia de presentación ligado al mayor número de muestras remitidas en intervalos de tiempo más cortos, 
Cuadro 1. Diagnósticos histopatológicos de neoplasias mamarias en caninos durante el periodo 2007 a 2016 en el Laboratorio de Histología, Embriología y Patología Animal (LHEPA) de la Facultad de Medicina Veterinaria (FMV) de la Universidad Nacional Mayor de San Marcos (UNMSM), Lima, con base a la clasificación de Goldschmidt et al. (2011)

\begin{tabular}{llcc}
\hline Tipo & Diagnóstico histopatológico & Número & Porcentaje \\
\hline \multirow{3}{*}{ Neoplasias } & Carcinoma tubular & 81 & 14.2 \\
& Carcinoma tubulopapilar & 31 & 5.4 \\
& Carcinoma papilar quístico & 244 & 42.8 \\
& Carcinoma sólido & 39 & 6.8 \\
& Comedocarcinoma & 2 & 0.4 \\
& Carcinoma tipo complejo & 23 & 4.0 \\
& Carcinoma tipo mixto & 39 & 6.8 \\
& Carcinoma ductal & 1 & 0.2 \\
& Carcinoma de células escamosas & 8 & 1.4 \\
& Condrosarcoma & 1 & 0.2 \\
& Fibrosarcoma & 12 & 2.1 \\
& Hemangiosarcoma & 9 & 1.6 \\
& Leiomiosarcoma & 4 & 0.7 \\
& Liposarcoma & 1 & 0.2 \\
& Mixosarcoma mamario & 2 & 0.4 \\
& Osteosarcoma & 1 & 0.2 \\
& Rabdomiosarcoma & 3 & 0.5 \\
Neoplasias & Tumor mamario mixto maligno & 39 & 6.8 \\
benignas & Adenoma simple & 5 & 0.9 \\
& Fibroadenoma & 6 & 1.1 \\
& Lipoma & 2 & 0.4 \\
& Hemangioma & 7 & 1.2 \\
\hline Total & Tumor mamario mixto benigno & 10 & 1.8 \\
\hline
\end{tabular}

posiblemente ante una mayor concientización de los dueños de las mascotas y la mayor expectativa de vida de los canes. Estudios realizados en México y Colombia reportan resultados similares respecto al porcentaje de neoplasias mamarias. Salas (2015) encontró $16.8 \%$ y Caicedo et al. (2012) reportó 17.8\% de frecuencia de neoplasias mamarias, respectivamente; sin embargo, Briceño (2014) en Venezuela registró $33.8 \%$.
Se reconoce que los tumores mamarios son el tipo neoplásico más frecuente en la hembra canina, los cuales están asociados a los ciclos estrales, cuyos cambios hormonales repercuten en el crecimiento del tejido mamario. El 98\% de neoplasias mamarias en hembras en este estudio coincide con e1 97\% reportado por Sánchez y Guarín (2014), el 99\% según Rostami y Tate (1994), y el 94\% de Chau et al. (2013). 


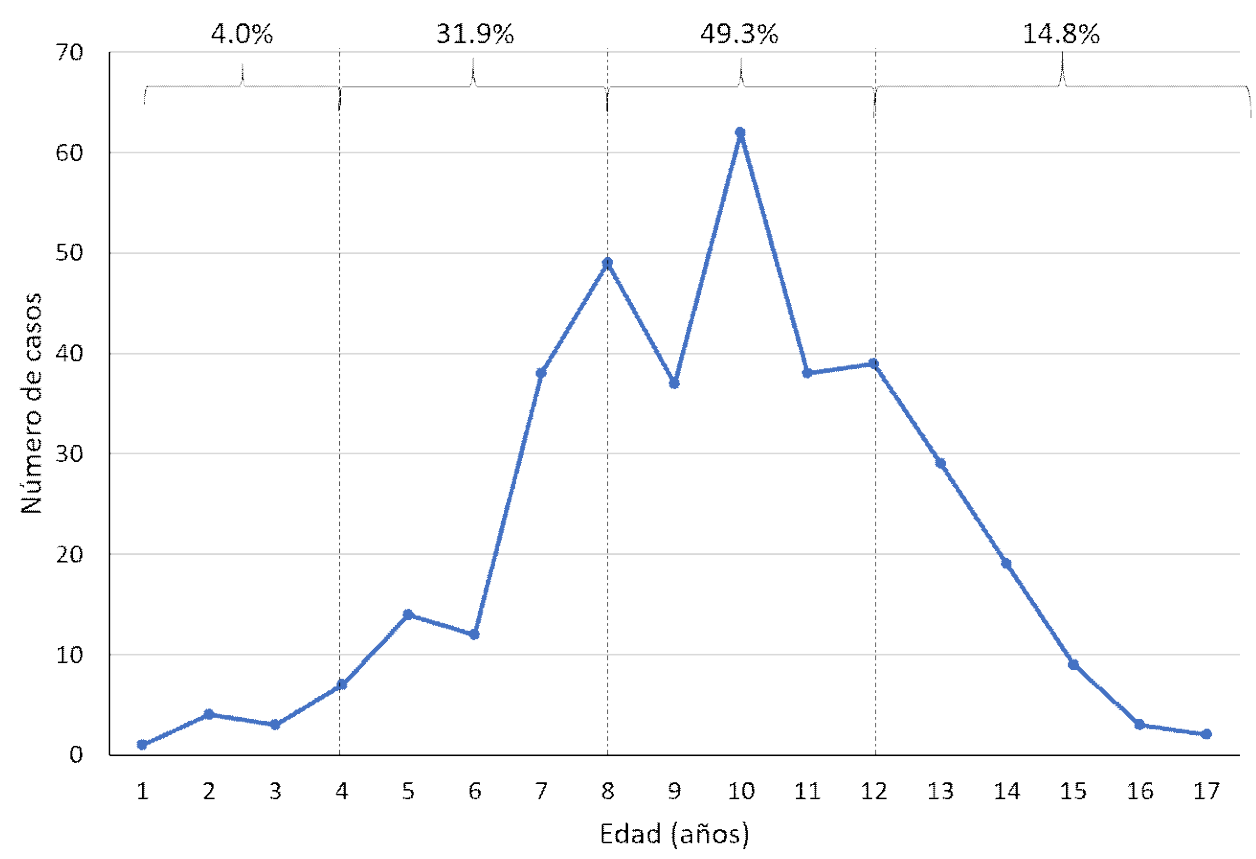

Figura 1. Presentación de neoplasias mamarias en caninos según edad, diagnosticadas histológicamente durante el periodo 2007-2016 en el Laboraorio de Histología, Embriología y Patología Animal (LHEPA) de la Facultad de Medicina Veterinaria (FMV) de la Universidad Nacional Mayor de San Marcos (UNMSM), Lima

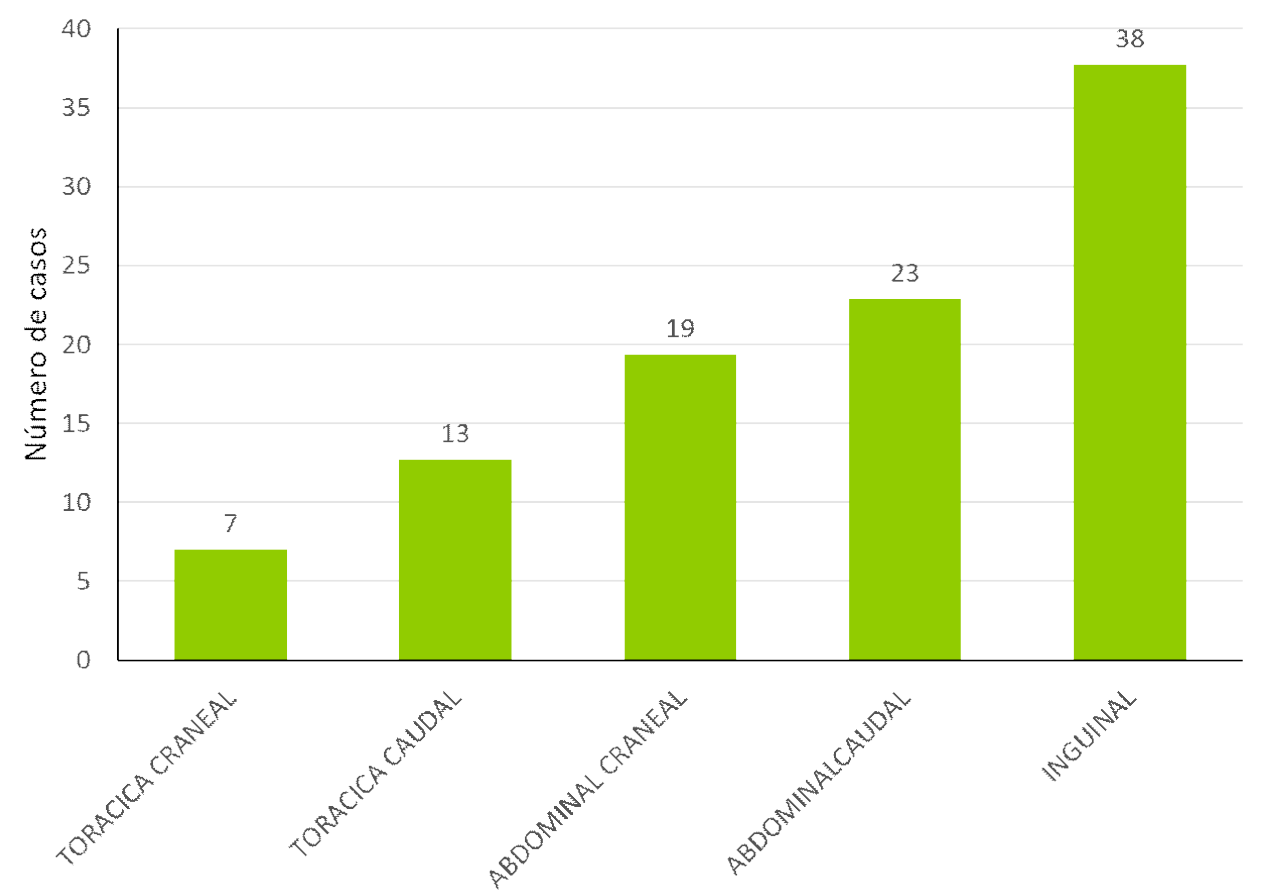

Figura 2. Localización de neoplasias de glándula mamaria en caninos registrados en el periodo entre 2007 y 2016 en el Laboratorio de Histología, Embriología y Patología Animal (LHEPA) de la Facultad de Medicina Veterinaria (FMV) de la Universidad Nacional Mayor de San Marcos (UNMSM), Lima 
Jubb et al. (2007) señala que $68 \%$ de 364 pacientes estudiados pertenecían a razas puras, mientras que el $32 \%$ era de origen mestizo. En el presente estudio, el grupo racial con mayor frecuencia de neoplasias mamarias estuvo representado por los perros mestizos (32\%) y la raza Cocker Spaniel $(13 \%)$. Por otro lado, el orden de las razas con mayor frecuencia de casos en este estudio fue similar a estudios previos en el país realizados por Chau et al. (2013) en el intervalo 1994-2006 y por Orbegoso (1993) entre 1973 y 1992. En México y Venezuela, las razas notificadas con mayor frecuencia incluyen mestizos, Poodle, Cocker (Briceño, 2014; Salas et al., 2015) y en Colombia fueron el Poodle, mestizos y Cocker (Caicedo et al., 2012).

De acuerdo con el comportamiento histopatológico indicado por Goldschmidt et al. (2011), las neoplasias malignas fueron las más frecuentes $(94.7 \%)$. En el Perú, Chau et al. (2013), por otro lado, obtuvo 78\%, Caicedo (2012) 80\%, mientras que Stratmann et al. (2008) reportó 74\%. Sin embargo, Gerosa (2007), Dobson y Duncan (2014), Salas et al. (2015) y Sánchez y Guarín (2014) registraron cerca del $50 \%$, mientras que Bronden et al. (2010) reportó 38\% de neoplasias mamarias malignas en Dinamarca. Es de resaltar que la presentación de tumores mamarios malignos es bastante alta en el país y se debería evaluar si se debe a sesgos por el mayor envío de muestras sospechosas de malignidad (Gerosa, 2007), por la decisión de los dueños de no aceptar la intervención quirúrgica o de no realizar el estudio histopatológico.

La variable de edad es resaltante en las neoplasias mamarias caninas. Diversos estudios han identificado que la edad de presentación con mayor número de casos se encuentra en caninos entre 7 y 13 años (Sánchez y Guarín, 2014). La mayor presentación de casos en este estudio se encontró entre los 9 y 12 años, descendiendo en canes de mayor edad, posiblemente por el menor número de canes gerontes (Gerosa, 2007;
Withrow y Vail, 2009), o por la preocupación de los dueños de someter a cirugía a caninos de edad avanzada. La mayor prevalencia de tumores mamarios caninos se observó en caninos de 10 años (17.7\%), como lo indica Gerosa (2007), aunque Dobson y Duncan (2014) señalan que la edad media de presentación es de 8 años.

Diversos estudios indican que el $60 \%$ de los tumores mamarios se localizan en la cuarta y quinta glándula mamaria (Chau et al., 2013; Ruano, 2013), lo cual coincide con el presente estudio con el $60.6 \%$ de los tumores presente en estas dos glándulas mamarias, ocurriendo el $37.7 \%$ en la glándula mamaria inguinal y $22.9 \%$ en la glándula mamaria abdominal caudal, probablemente debido al mayor volumen de tejido mamario susceptible a la transformación tumoral (Gerosa, 2007; Sánchez y Guarín, 2014). Según Ruano (2013), más del 50\% de los casos de neoplasias mamarias fue de presentación múltiple; sin embargo, en este estudio solo el $35 \%$ de casos tuvo presentación múltiple, aunque esto pudo ser debido a falta de información brindada al llenar el formulario, ya que no se registró si el canino había sido sometido a cirugías previas.

El tipo histopatológico más frecuente en esta recategorización fue el carcinoma papilar quístico $(42.8 \%)$, seguido por el carcinoma tubular (13.9\%), lo cual se acerca a los resultados obtenidos por Briceño (2014) basados en la clasificación de Goldschmidt et al. (2011), en el que estos carcinomas ocuparon el segundo y tercer lugar, pero liderado por el carcinoma complejo. Las neoplasias mamarias benignas representaron el 5.3\%, especialmente el tumor mamario mixto benigno, hemangioma y fibroadenoma, lo cual difiere del estudio de Briceño (2014), quién encontró el adenoma simple y el adenoma intraductal como las neoplasias benignas más frecuentes. Múltiples diagnósticos no presentaron cambios respecto a la nomenclatura al realizar la recategorización; sin embargo, se reporta el diagnóstico de comedocarcinoma como neoplasias malignas de glándula 
mamaria que presentaron zonas de necrosis en el centro de los nidos formados por células epiteliales malignas.

\section{Conclusiones}

- La frecuencia de neoplasias mamarias en caninos fue del $22.4 \%$ del total de procesos neoplásicos diagnosticados en el periodo 2007-2016 en el Laboratorio de Histología, Embriología y Patología Animal de la Facultad de Medicina Veterinaria de la Universidad Nacional Mayor de San Marcos.

- Las neoplasias mamarias se presentaron con mayor frecuencia en perras de 8 a 12 años.

- El Cocker fue la raza más afectada.

- Las glándulas mamarias caudales fueron las más afectadas.

- La presentación única fue más frecuente frente a múltiples neoformaciones.

- La mayoría de las neoplasias mamarias fueron malignas y de acuerdo con la Clasificación de Goldschmidt et al. (2011), el diagnóstico histopatológico más frecuente fue el carcinoma papilar quístico (42.8\%).

\section{Literatura Citada}

1. Briceño A, Castillo C, Morales M. 2014. Estudio retrospectivo de la casuística de tumores mamarios en pequeños animales en un servicio privado de anatomía patológica veterinaria en Caracas-Venezuela. REDVET 17(10). [Internet]. Disponible en: http:// www.veterinaria.org/revistas/redvet/ n111116.html

2. Bronden LB, Nielsen SS, Toft N, Kristensen AT. 2010. Data from the Danish veterinary cancer registry on the occurrence and distribution of neoplasms in dogs in Denmark. Vet Rec 166: 586490. doi: 10.1136/vr.b4808
3. Caicedo JA, Iregui CA, Cabarcas ME, Acosta BJ. 2012. Estudio comparativo de la frecuencia de tumores mamarios según sexo, edad y tipo histológico en caninos y humanos en los laboratorios de patología anatómica de la Universidad Nacional de Colombia sede Bogotá. Rev Colomb Cienc Anim 5: 37-46.

4. Chau G, Chavera A, Perales $R$, Gavidia C. 2013. Frecuencia de neoplasias en glándula mamaria de caninos: estudio retrospectivo en el periodo 1992-2006 en la ciudad de Lima, Perú. Rev Inv Vet Perú 24: 72-77. doi: 10.15381/rivep.v24i1.1663

5. Dobson JM, Duncan LB. 2014. Manual de oncología en pequeños animales. $3^{\circ}$ ed. Barcelona: Ed. 540 p.

6. Gerosa RM. 2007. Geriatria canina. Buenos Aires: Intermedica. 688 p.

7. Goldschmidt M, Peña L, Rasotto R.2011. Classification and grading of canine mammary tumors. Vet Pathol 48: 117-131. doi: 10.1177/0300985810393258

8. Hampe JF, Misdorp W. 1974. IX. Tumours and dysplasias of the mammary gland. Bull World Health Organ 50: 111-133.

9. Jubb K, Kennedy P, Palmer N. 2007. Pathology of domestic animals. $5^{\circ} \mathrm{ed}$. Ontario: Saunders. $2340 \mathrm{p}$.

10. Misdorp W, Else RW, Hellman E, Lipscomb TP. 1999. Histologic classification of mammary tumors of the dog and the cat. Washington, USA: Armed Forces Institute of Pathology. $59 \mathrm{p}$.

11. Núñez L, de Oca A. 2012. Frecuencia de neoplasias de glándula mamaria en perras con diagnóstico citológico. Rev Cient-Fac Cien V 5: 426-431.

12. Orbegoso G. 1993. Neoplasias de glándula mamaria de caninos estudio estadístico de la FMV - UNMSM durante el periodo 1973-1992. Tesis de Médico Veterinario. Lima: Univ. Nacional Mayor de San Marcos. 34 p.

13. Rostami M, Tateyama S, Uchida K, Naitou H, Yamaguchi R, Otsuka H. 1994. Tumors in domestic animals 
examined during a ten year period 1980 1989) at Miyazaki University. J Vet Med 56:403-405.

14. Ruano R. 2013. Oncología práctica para el clínico de pequeños animales. Barcelona: Multimédica Ediciones Veterinarias. $366 \mathrm{p}$.

15. Salas Y, Márquez A, Diaz D, Romero L. 2015. Epidemiological study of mammary tumors in female dogs diagnosed during the period 2002-2012: a growing animal health problem. Plos One 10: e0127381. doi: 10.1371/ journal.pone. 0127381

16. Sánchez FR, Guarín CE. 2014. Tumores de glándula mamaria en caninos. Conexagro JDC 4: 60- 78.

17. Stratmann N, Failing K, Richter A, Wehrend A. 2008. Mammary tumor recurrence in bitches after regional mastectomy. Vet Surg 37:82-86. doi: 10.1111/j.1532-950X.2007.00351.x.

18. Withrow S, Vail D. 2009. Withrow and Mac Even's small animal clinical oncology. $4^{\circ}$ ed. Barcelona: Multimédica Ediciones Veterinarias. 768 p. 